\title{
Predictions for neutrino structure functions
}

\author{
S. Kretzer \\ Institut für Physik, Universität Dortmund, Dept. of Physics, D-44227 Dortmund, Germany \\ and Department of Physics and Astronomy, Michigan State University, East Lansing, Michigan 48824-1116 \\ F. I. Olness and R. J. Scalise \\ Department of Physics, Southern Methodist University, Dallas, Texas 75275-0175 \\ R. S. Thorne \\ Cavendish Laboratory, University of Cambridge, Madingley Road, Cambridge, CB3 OHE, United Kingdom \\ U. K. Yang \\ University of Chicago, Enrico Fermi Institute, Chicago, Illinois 60637-1434 \\ and University of Rochester, Rochester, New York 14627 \\ (Received 19 January 2001; published 3 July 2001)
}

\begin{abstract}
The first measurements of $\Delta x F_{3}$ are higher than current theoretical predictions. We investigate the sensitivity of these theoretical predictions upon a variety of factors including the renormalization scheme and scale, quark mass effects, higher twist, isospin violation, and PDF uncertainties.
\end{abstract}

DOI: 10.1103/PhysRevD.64.033003

PACS number(s): 13.15.+g, 12.38.Qk, 24.80.+y, 25.30.-c

\section{INTRODUCTION}

Deep inelastic lepton-nucleon (DIS) scattering experiments have provided precision information about the quark distributions in the nucleon. However, there has been a longstanding discrepancy between the $F_{2}$ structure functions extracted from neutrino and muon experiments in the small $x$ range. Recently, a new analysis of differential cross sections and structure functions from CCFR $\nu_{\mu}$-Fe and $\bar{\nu}_{\mu}$-Fe data was presented; in this study, the neutrino-muon difference is resolved in part by extracting the $\nu_{\mu}$ structure functions in a physics model independent way [1].

In previous analyses of $\nu_{\mu}$ data [2], structure functions were extracted by applying a slow rescaling correction to correct for the charm mass suppression in the final state. In addition, the $\Delta x F_{3}$ term (used as input in the extraction) was calculated from a leading order charm production model. These resulted in physics model dependent (PMD) structure functions. In the new analysis [1], slow rescaling corrections are not applied, and $\Delta x F_{3}$ and $F_{2}$ were extracted from two parameter fits to the data.

The extracted physics model independent (PMI) values for $F_{2}^{\nu}$ are then compared with $F_{2}^{\mu}$ within the framework of next leading order (NLO) models for massive charm production; these are found to be in agreement, thus resolving the long-standing discrepancy between the two sets of data. ${ }^{1}$ However, the first measurements of $\Delta x F_{3}$ are systematically higher than current theoretical predictions. The objective of

\footnotetext{
${ }^{1}$ A comparison of $F_{2}$ from neutrino DIS to that from charged lepton DIS shows good agreement above $x=0.0125$, but shows differences at smaller $x$. This low $x$ discrepancy can be explained by the different behavior of $F_{2}$ from $\nu$ DIS to that from $e / \mu$ DIS as $Q^{2} \rightarrow 0$. See Ref. [3] for details.
}

this paper is to investigate the sensitivity of $\Delta x F_{3}$ upon a variety of factors including renormalization scheme and scale, quark mass effects, higher twist, isospin violation, and parton distribution functions (PDF) uncertainties.

In Fig. 1 we have plotted the quantity $\Delta x F_{3}$ for an isoscalar target computed to order $\alpha_{s}^{1}$. For comparison, we also display data from the CCFR analysis. [1] We observe the trend that the theory lies systematically about $1 \sigma$ below the data; while there is much freedom in the theoretical calculation, the difference between these calculations and the data at low $Q$ values warrants further investigation. We will discuss and compare the different theoretical calculations, and examine the inherent uncertainty in each with respect to different input parameters. We will also examine the experimental input, and assess uncertainties in this data.

We will generally display $\Delta x F_{3}$ as a function of $Q^{2}$. For completeness, in Fig. 2 we display $\Delta x F_{3}$ vs $x$ for four values of $Q^{2}(\mathrm{GeV})$. These curves are in the Aivazis-CollinsOlness-Tung (ACOT) scheme using CTEQ4HQ with $\mu=Q$. The behavior of the other schemes is similar.

\section{A. Measurement of $\Delta x F_{3}$}

The structure functions are defined in terms of the neutrino-nucleon cross section via

$$
\begin{aligned}
\frac{d^{2} \sigma^{\nu, \bar{\nu}}}{d x d y}= & \frac{G_{F}^{2} M E_{\nu}}{\pi}\left[F_{2}(1-y)+x F_{1} y^{2} \pm x F_{3} y\left(1-\frac{y}{2}\right)\right] \\
= & \frac{G_{F}^{2} M E_{\nu}}{\pi}\left[F_{2}\left\{(1-y)+\frac{y^{2} 1+4 M^{2} x^{2} / Q^{2}}{21+R\left(x, Q^{2}\right)}\right\}\right. \\
& \left. \pm x F_{3} y\left(1-\frac{y}{2}\right)\right]
\end{aligned}
$$

where $G_{F}$ is the Fermi weak coupling constant, $M$ is the nucleon mass, $E_{\nu}$ is the incident energy, $y=E_{h} / E_{\nu}$ is the 

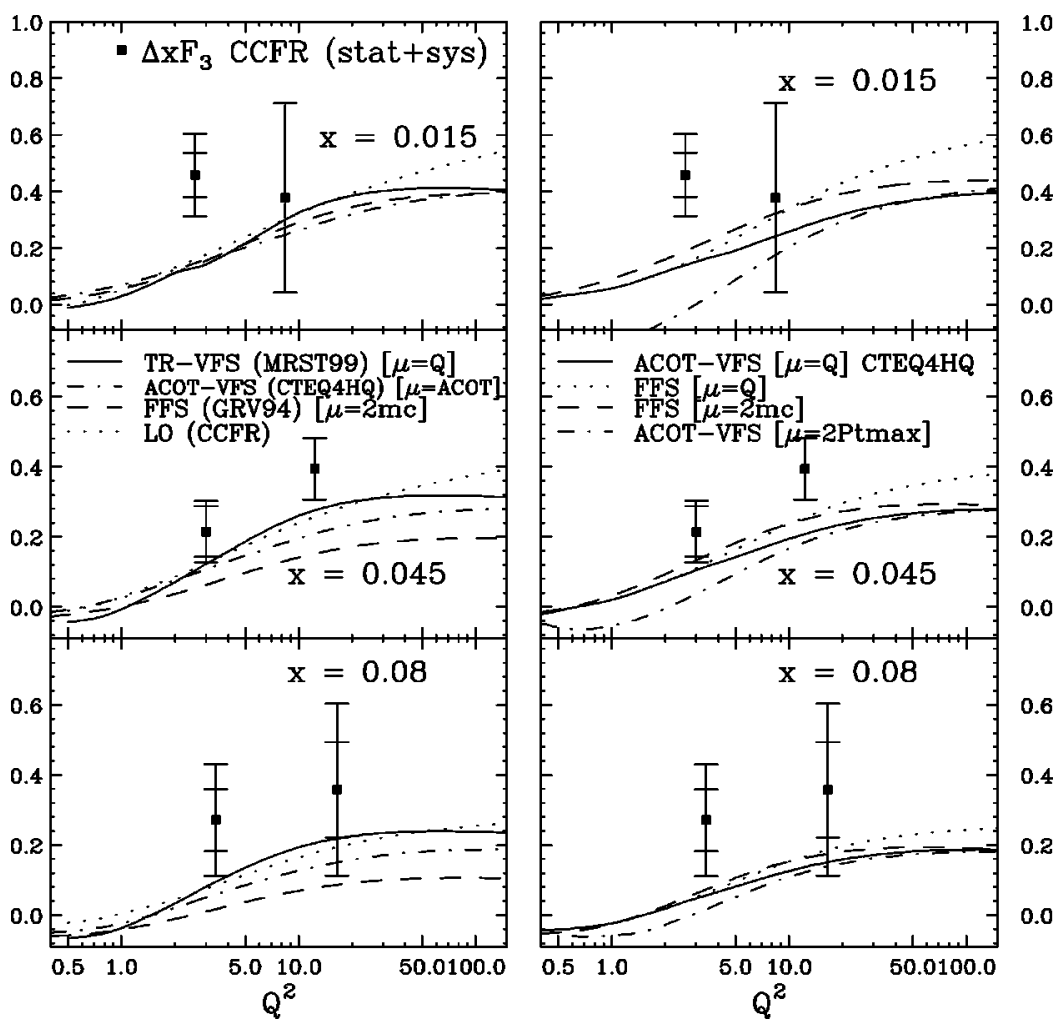

FIG. 1. Comparison of $\Delta x F_{3}$ data from CCFR with various theoretical predictions. fractional energy transfer, and $E_{h}$ is the final state hadronic energy. In the second form of the above equation, we have used the relation

$$
2 x F_{1}\left(x, Q^{2}\right)=F_{2}\left(x, Q^{2}\right) \frac{1+4 M^{2} x^{2} / Q^{2}}{1+R\left(x, Q^{2}\right)}
$$

where $R=\sigma_{L} / \sigma_{T}$ is the ratio of the cross sections of longitudinally- to transversely-polarized $W$-bosons, $Q^{2}$ is the square of the four-momentum transfer to the nucleon, and $x=Q^{2} / 2 M E_{h}$ is the Bjorken scaling variable. For $x<0.1, R$ in neutrino scattering is expected to be somewhat larger than $R$ for muon scattering because of the production of massive charm quarks in the final state for the charged current neutrino production.

The sum of $\nu_{\mu}$ and $\bar{\nu}_{\mu}$ differential cross sections for charged current interactions on an isoscalar target is then

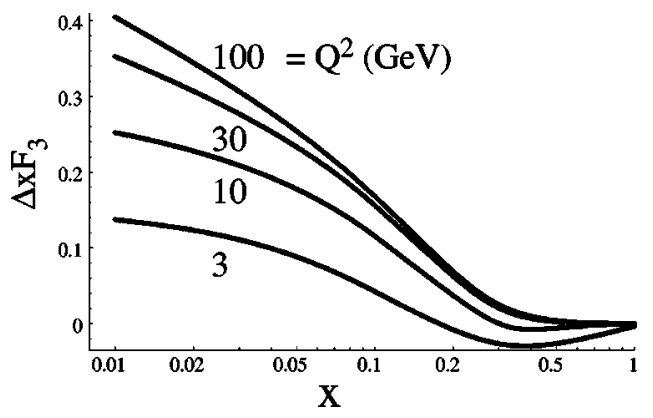

FIG. 2. $\Delta x F_{3}$ vs $x$ for four values of $Q^{2}\left(\mathrm{GeV}^{2}\right)$ in the ACOT scheme using CTEQ4HQ with $\mu=Q$.

$$
\begin{aligned}
F(\boldsymbol{\epsilon}) & \equiv\left[\frac{d^{2} \sigma^{\nu}}{d x d y}+\frac{d^{2} \sigma^{\bar{\nu}}}{d x d y}\right] \frac{(1-\epsilon) \pi}{y^{2} G_{F}^{2} M E_{\nu}} \\
& =2 x F_{1}[1+\epsilon R]+\frac{y(1-y / 2)}{1+(1-y)^{2}} \Delta x F_{3}
\end{aligned}
$$

where $\epsilon \simeq 2(1-y) /\left[1+(1-y)^{2}\right]$ is the polarization of the virtual $W$ boson.

Using Eq. (3) and Eq. (2), $F_{2}$ and $\Delta x F_{3}$ can be extracted separately. Because of the positive $\mathrm{e}^{2}$ correlation between $R$ and $\Delta x F_{3}$, the extracted values of $F_{2}$ are rather insensitive to the input $R$. If a large input $R$ is used, a larger value of $x F_{3}$ is extracted from the $y$ distribution, thus yielding the same value of $F_{2}$. In contrast, the extracted values of $\Delta x F_{3}$ are sensitive to the assumed value of $R$, which yields a larger systematic error, shown on the data. For $R$ the QCD-inspired fit in [4] is used, but corrected for charged current neutrino scattering using a leading order slow rescaling model. This gives precisely the same type of correction as a full NLO calculation including the massive charm quark, as shown in Ref. [5], but leads to a somewhat higher normalization than the perturbative correction. It is arguable which prescription for $R$ leads to the better fit to existing data, but the difference between the two generates a significant fraction of the systematic error shown. Clearly a further reduction in the assumed value of $R$ (even down to zero), as suggested by models including $\ln (1 / x)$ resummations, would still leave a

\footnotetext{
${ }^{2}$ This correlation is best observed in the second form of Eq. (1).
} 
discrepancy between the lowest $Q^{2}$ data points and the theoretical predictions.

\section{B. Quark parton model relations}

Now that we have outlined the experimental method used in the extraction of the $F$ structure functions, it is instructive to recall the simple leading-order correspondence between the F's and the PDF's: ${ }^{3}$

$$
\begin{gathered}
F_{2}^{(\nu, \bar{v}) N} \simeq x\{u+\bar{u}+d+\bar{d}+s+\bar{s}+c+\bar{c}\} \\
x F_{3}^{(\nu, \bar{v}) N} \simeq x\{u-\bar{u}+d-\bar{d} \pm(s+\bar{s}) \mp(c+\bar{c})\} .
\end{gathered}
$$

Therefore, the combination $\Delta x F_{3}$ yields [6]

$$
\Delta x F_{3}=x F_{3}^{\nu N}-x F_{3}^{\bar{\nu} N} \simeq 2 x\{(s+\bar{s})-(c+\bar{c})\} .
$$

Note that since this quantity involves the parity violating structure function $F_{3}$, this measurement has no analogue in the neutral current photon-exchange process. Also note that since, at leading-order, $\Delta x F_{3}$ is directly sensitive to the strange and charm distributions, this observable can be used to probe the heavy quark PDF's, and to understand heavy quark (charm) production. We discuss these possibilities further in the following subsection.

\section{Implications for PDF's}

We have illustrated in Eq. (5) how $\Delta x F_{3}$ is closely tied to the heavy quark PDF's. The question is: given the present knowledge base, should we use $\Delta x F_{3}$ to determine the heavy quark PDF'S, or vice versa? To answer this question, we briefly review present measurements of heavy quark PDF's, and assess their uncertainty.

\section{Tevatron $W+Q$ production}

The precise measurement of $W$ plus heavy quark ( $W$ $+Q$ ) events provides important information on heavy quark PDF's; additionally, such signals are a background for Higgs boson and squark searches $[7,8]$.

Unfortunately, a primary uncertainty for $W+Q$ production comes from the heavy quark PDF's. Given that $\Delta x F_{3}$ is sensitive to these heavy quark PDF's, we see at least two scenarios. One possibility is that new analysis of present data will resolve this situation prior to run II, and provide precise distributions as an input to the Tevatron data analysis. If the situation remains unresolved, then new data from run II may help to finally solve this puzzle. In the future, a neutrino experiment from a high intensity proton facility or from a neutrino factory at a muon storage ring would be an ideal tool to measure any neutrino structure function [9].

\footnotetext{
${ }^{3}$ To exhibit the basic structure, the above is taken in the limit of 4 quarks, a symmetric sea, and a vanishing Cabibbo angle. Of course, the actual analysis takes into account the full structure. [1]
}
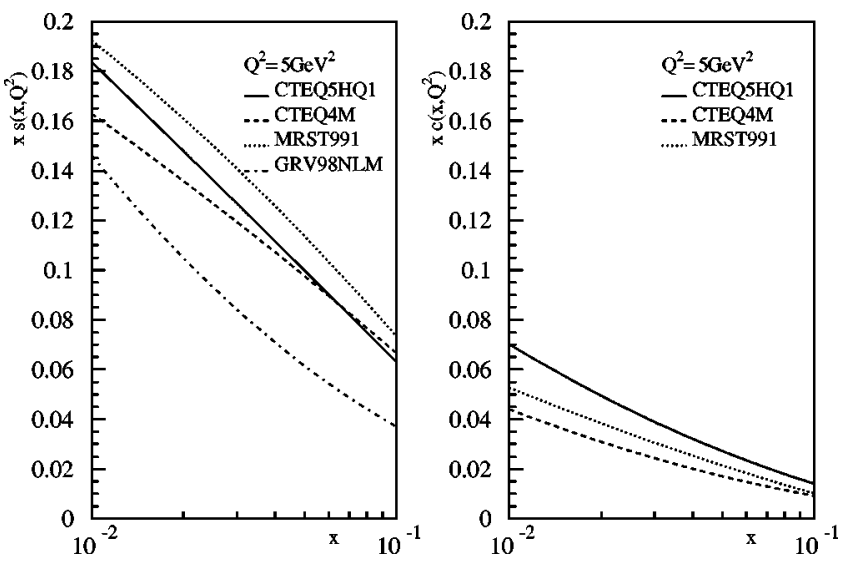

FIG. 3. (a) We display several strange sea PDFs in the relevant $x$ range at $Q^{2}=5 \mathrm{GeV}$. (b) Same as above for the charm sea. GRV uses the fixed-flavor-scheme (FFS) with 3 flavors, so there is no partonic charm in these PDFs.

\section{DIS di-muon production}

The strange distribution is directly measured by dimuon production in neutrino-nucleon scattering. ${ }^{4}$ The basic subprocess is $\nu s \rightarrow \mu^{-} c X$ with a subsequent charm decay $c$ $\rightarrow \mu^{+} X^{\prime}$.

DIS dimuon measurements have safely established the breaking of the $S U(3)$ flavor symmetry,

$$
\left.\kappa \equiv \frac{\int d x x s(x)}{\int d x x[\bar{u}(x)+\bar{d}(x)] / 2}\right|_{Q^{2}=\mathcal{O}\left(10 \mathrm{GeV}^{2}\right)} \lesssim \frac{1}{2}
$$

in the nucleon sea. Still, there remain large uncertainties for the $s$-quark distribution in the kinematic regions relevant for $\Delta x F_{3}$, even though the $(x, Q)$-range of the CCFR dimuon measurements $[11,15]$ is comparable. CCFR recorded 5044 $\nu_{\mu}$ and $1062 \bar{\nu}_{\mu} \mu^{\mp} \mu^{ \pm}$events with $P_{\mu_{1}} \geqslant 9 \mathrm{GeV}, P_{\mu_{2}} \geqslant 5$ $\mathrm{GeV}, 30 \leqslant E_{\nu} \leqslant 600 \mathrm{GeV},\left\langle Q^{2}\right\rangle=22.2 \mathrm{GeV}^{2}$, and $0.01 \leqslant x$ $\leqslant 0.4$. The more recent $\mathrm{NuTeV}$ experiment recorded a similar sample of events [16], and these are presently being analyzed through a MC simulation based on NLO quark- and gluoninitiated corrections at differential level $[12,17,18]$. A complete NLO analysis of this data together with a global analysis may help to further constraint the strange quark distribution.

At present, PDF sets take strangeness suppression into account by imposing the constraint in Eq. (6) as $s(x)$ $=\kappa[\bar{u}(x)+\bar{d}(x)] / 2$ at the PDF input scale $Q_{0} \simeq 1 \mathrm{GeV}$, $[19,20]$ or by evolving $s(x)$ from a vanishing input at a lower scale [21]. The residual uncertainty can be large, as can be seen from the collection of strange seas in Fig. 3.

\footnotetext{
${ }^{4}$ Presently, there are a number of LO analyses, and one NLO ACOT analysis [10-13]. Results of a recent LO analysis by NOMAD [14] are in line with these experiments.
} 
In the following, we will take the uncertainty in the strange PDF into account by relaxing the experimental constraint in Eq. (6) as implemented in the Martin-RobertsStirling-Thorne (MRST) partons [19]. This will be discussed in Sec. III B.

\section{Charged and neutral current DIS}

The strange distribution can also be extracted indirectly using a combination of charged $\left(F_{2}^{\nu}\right)$ and neutral $\left(F_{2}^{\mu}\right)$ current structure functions; however, the systematic uncertainties involved in this procedure make an accurate determination difficult [1]. The basic idea is to use the (leading-order) relation

$$
\frac{F_{2}^{\mu}}{F_{2}^{\nu}} \simeq \frac{5}{18}\left\{1-\frac{3(s+\bar{s})-(c+\bar{c})+\cdots}{q+\bar{q}}\right\}
$$

to extract the strange distribution. Here, $q+\bar{q}$ represents a sum over all quark flavors. This method is complicated by a number of issues including the $x F_{3}$ component which can play a crucial role in the small- $x$ region-precisely the region where we observe the discrepancy. From the corresponding relation

$$
\frac{5}{18} F_{2}^{\nu}-F_{2}^{\mu} \simeq \frac{1}{12} \Delta x F_{3}
$$

we see that these problems are not independent; however, this information, together with the exclusive dimuon events, may provide a more precise determination of the strange quark sea, and help to resolve our puzzle.

Prior to the DIS dimuon data, the 1992 CTEQ1 analysis [22] found that a combination of neutral current (NC) structure functions from the New Muon Collaboration (NMC) [23] and the physics-model-dependent charged current structure functions from CCFR [11] seemed compatible with approximate $S U(3)$ symmetry, i.e., $\kappa \sim 1$ in Eq. (6). Recent dimuon measurements now exclude an $S U(3)$ symmetry $\kappa$ $=1$.

We shall explore the effect of $\kappa$ on $\Delta x F_{3}$ in Sec. III B.

\section{DEPENDENCE OF $\Delta x F_{3}$ ON INPUT PARAMETERS}

We now systematically investigate the sensitivity of the theoretical predictions of $\Delta x F_{3}$ upon a variety of factors including: renormalization scheme and scale, quark mass effects, higher twist, isospin violation, and PDF uncertainties.

To simplify this analysis, we first examine the influence of these factors on the LO expression: $\Delta x F_{3}=2 x\{[s(\xi, \mu)$ $+\bar{s}(\xi, \mu)]-[c(\xi, \mu)+\bar{c}(\xi, \mu)]\} \simeq 4 x\{s(\xi, \mu)-c(x, \mu)\}$ after using this as a "toy model," we will then return to the full NLO calculation in the next section. ${ }^{5}$ For most variables, the

\footnotetext{
${ }^{5}$ Note that $\Delta x F_{3}$ only depends on the average value of the seaquark distributions; any differences due to a possible $s \neq \bar{s}$ or $c \neq \bar{c}$ asymmetry would average out [24].
}
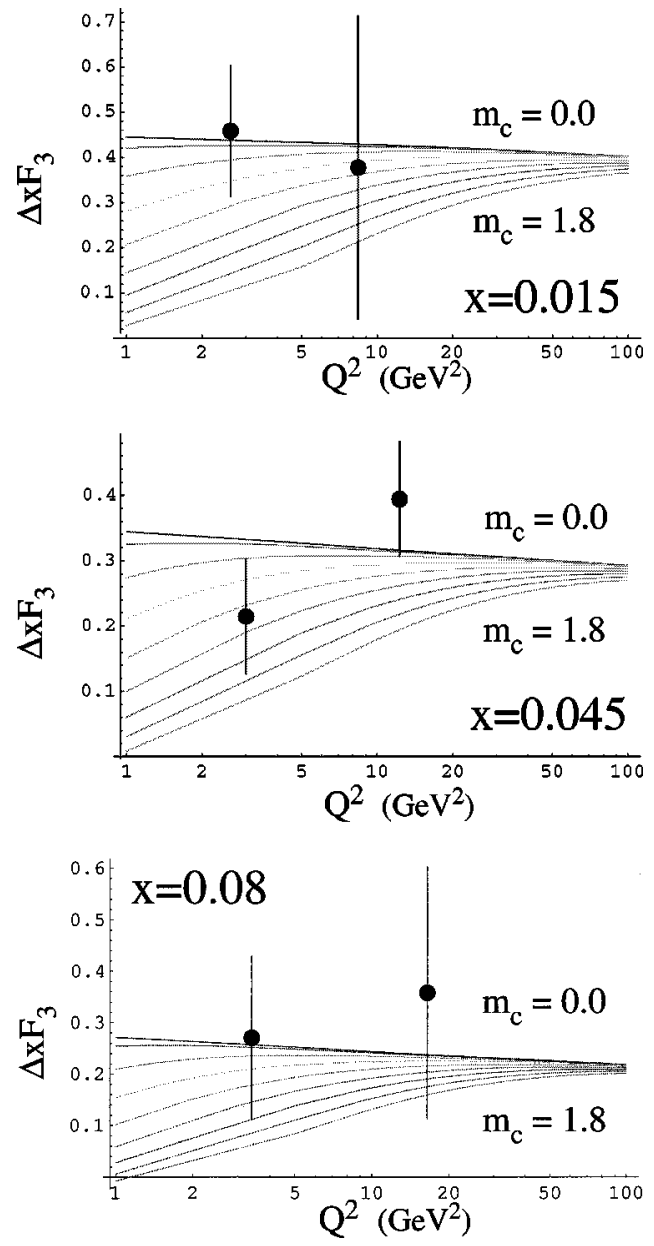

FIG. 4. Variation of the leading-order expression $\Delta x F_{3}$ $\simeq 4 x\{s(\xi, \mu)-c(x, \mu)\}$ on the charm mass, $m_{c}$, plotted vs $\mu$. We take values of $m_{c}$ in the range $m_{c}=[0,1.8] \mathrm{GeV}$ using steps of 0.2 $\mathrm{GeV}$ for three choices of $x$. We use $\xi=x\left(1+m_{c}^{2} / Q^{2}\right)$, and $\mu^{2}$ $=\left(Q^{2}+1.6^{2} \mathrm{GeV}^{2}\right)$.

simplified LO is sufficient to display the general behavior of the full NLO result. There are two exceptions: (1) the scheme dependence, and (2) the PDF dependence. These factors depend on the interplay of both the quark-initiated LO contributions as well as the NLO gluon-initiated contributions. For this reason, we will postpone discussion of these effects until the following section.

\section{A. Charm mass}

We start by examining the effect of the charm mass, $m_{c}$, on $\Delta x F_{3}$. In Fig. 4, we plot the leading-order expression $\Delta x F_{3} \simeq 4 x\{s(\xi, \mu)-c(x, \mu)\}$ vs $Q^{2}$ for values of $m_{c}$ in the range $=[0,1.8] \mathrm{GeV}$ using steps of $0.2 \mathrm{GeV}$. Here, we define $\xi=x\left(1+m_{c}^{2} / Q^{2}\right)$ which is a "slow-rescaling" type of correction $[25,26]$ which (crudely) includes mass effects by shifting the $x$ variable. "Slow rescaling" naturally arises at LO from the single-charm threshold condition $\left(W>m_{c}\right)$ and is required at NLO for consistent mass factorization [26,27].

Note that the result of this correction is most significant at low $Q^{2}$. To isolate the kinematic $m_{c}$ dependence, we have 

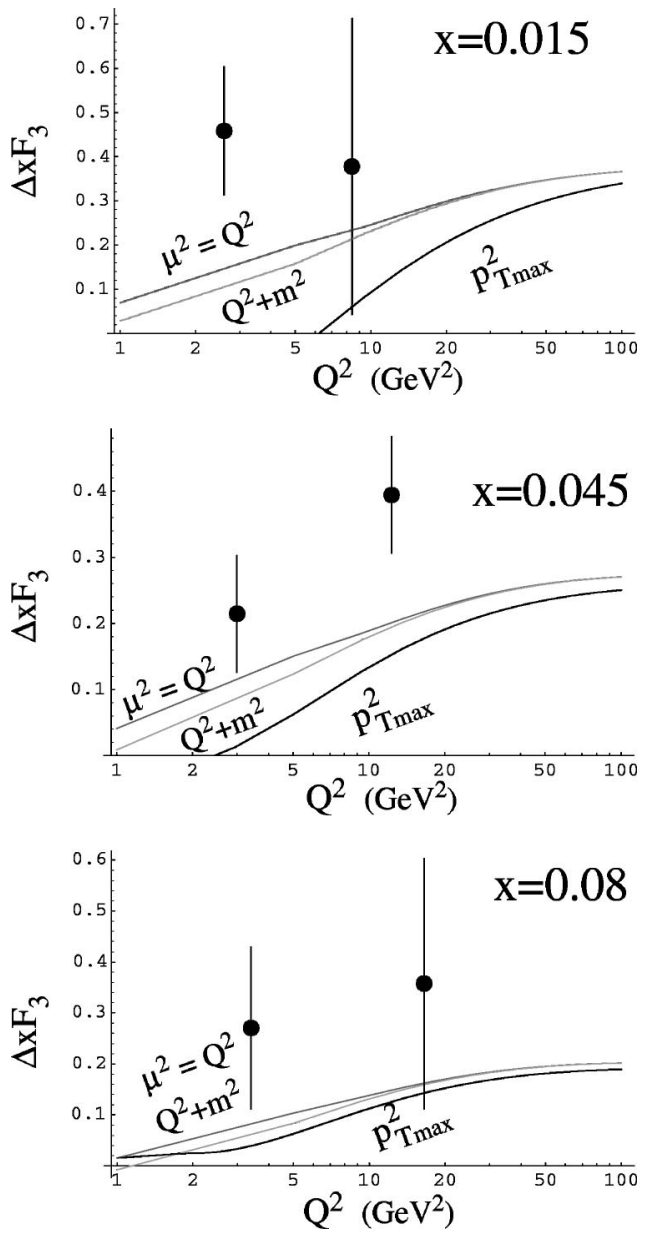

FIG. 5. Variation of the leading-order $\Delta x F_{3}$ on the renormalization scale, $\mu$. We have chosen $\mu^{2}=\left\{Q^{2}, Q^{2}+m_{c}^{2}, P_{T_{\max }}^{2}\right\}$.

chosen the scale $\mu^{2}=Q^{2}+1.6^{2} \mathrm{GeV}^{2}$. (We will separately vary the scale in the following subsection.)

Note that this exercise is only altering the charm mass in one aspect of the calculation; to be entirely consistent it would be necessary to obtain parton distributions (particularly the charm quark) using fits with different charm masses. For charm masses in the range 1.2 to $1.8 \mathrm{GeV}$, this will be a small effect; for charm masses below $\sim 1.2 \mathrm{GeV}$ (which is below the experimentally allowed range $[28,29]$ ), such issues become important and the curves of Fig. 5 will be modified, i.e. lower $m_{c}$ will lead to longer evolution for charm, a larger charm distribution, and a lowering of the curves in Fig. 5.

While taking $m_{c} \rightarrow 0$ does raise the theoretical curves in the regions where we observe the discrepancy (namely, the low $Q$ region), varying $m_{c}$ even within the wide range of $[1.2,1.8] \mathrm{GeV}$ (lower 4 curves) does not give us sufficient flexibility to match either the shape or normalization of the data.

\section{B. $\mu$ scale}

Next, we investigate the variation of $\Delta x F_{3} \simeq 4 x\{s(\xi, \mu)$ $-c(x, \mu)\}$ with the renormalization-factorization scale $\mu$. We use three choices of the $\mu^{2}$ scale:
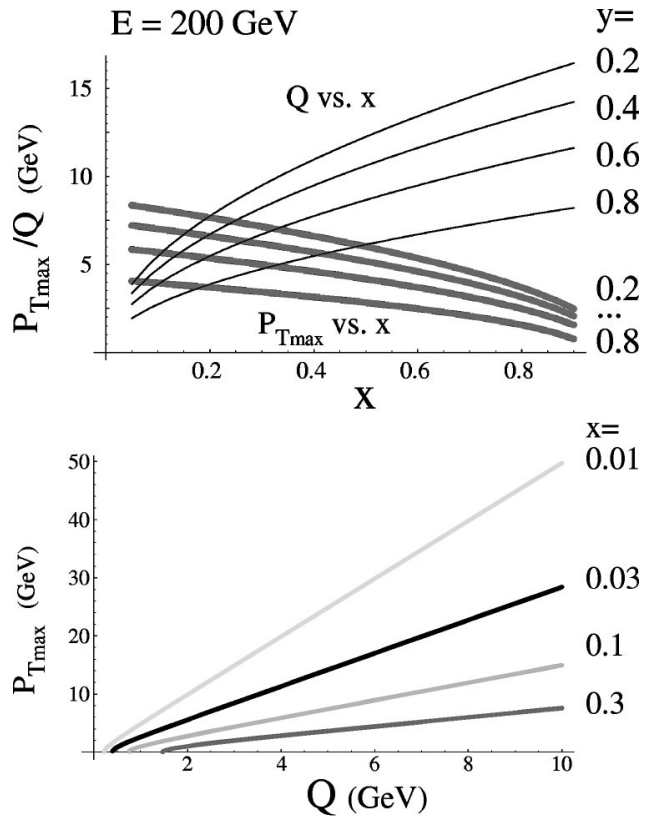

FIG. 6. (a) We display $P_{T}^{\max }$ and $Q$ vs $x$ for $\mathrm{E}=200 \mathrm{GeV}$ and $y=\{0.2,0.4,0.6,0.8\}$. (b) $P_{T}^{\max }$ vs $Q$ for $x=\{0.3,0.1,0.03,0.01\}$.

$$
\begin{gathered}
\mu^{2}=Q^{2}, \\
\mu^{2}=Q^{2}+m_{c}^{2}, \\
\mu^{2}=P_{T_{\max }^{2}}^{2}
\end{gathered}
$$

Of course, $\Delta x F_{3}$ with $\mu^{2}$ scales of $Q^{2}$ and $Q^{2}+m_{c}^{2}$ differ only at lower values of $Q^{2} ; \Delta x F_{3}$ with $\mu^{2}$ scale of $P_{T_{\max }}^{2}$ is comparable to $Q^{2}$ and $Q^{2}+m_{c}^{2}$ at larger $x$, but lies below for smaller $x$. The scale choice $Q^{2}+m_{c}^{2}$ leads to an improvement over $Q^{2}$ by providing a lower bound on $\mu$ to keep the scale in the perturbative region.

The choice of $P_{T_{\max }}$ is motivated, in part, by some observations by Collins [30]. To display the relationship between $P_{T_{\max }}$ and $Q$, in Fig. 6 we plot both $P_{T_{\max }}$ and $Q$ vs $x$ for 4 choices of $y$; note [15] that the $x$-dependence of $P_{T_{\max }}$ is opposite that of $Q$. While tying the scale choice of $\mu$ to $P_{T_{\max }}$ has some interesting intuitive interpretations, ${ }^{6}$ for small $x$ this scale clearly becomes too large for the relevant physics. In any case, it cannot help us with our $\Delta x F 3$ problem as the scale choice of $P_{T_{\max }}$ moves the theory curves away from the data.

\section{Higher twist}

We now illustrate the potential effects due to higher twist contributions. We parametrize such contributions by multiplying the leading-twist terms by a correction factor

\footnotetext{
${ }^{6}$ Technically, the interpretation is in terms of the characteristic $P_{T}$ of the partonic subprocess, but this is unobservable; therefore $P_{T_{\max }}$ is used instead [30].
} 
TABLE I. Values of the higher-twist coefficient $D_{2}(x)$ extracted from the LO, NLO and NNLO fits. Units are $\mathrm{GeV}^{2}$. Table taken from Ref. [51].

\begin{tabular}{lccc}
\hline \hline$x$ & LO & NLO & NNLO \\
\hline $0-0.0005$ & -0.4754 & 0.0116 & -0.0061 \\
$0.0005-0.005$ & -0.2512 & -0.0475 & 0.0437 \\
$0.005-0.01$ & -0.2481 & -0.1376 & -0.0048 \\
$0.01-0.06$ & -0.2306 & -0.1271 & -0.0359 \\
$0.06-0.1$ & -0.1373 & -0.0321 & 0.0167 \\
$0.1-0.2$ & -0.1263 & -0.0361 & 0.0075 \\
$0.2-0.3$ & -0.1210 & -0.0893 & -0.0201 \\
$0.3-0.4$ & -0.0909 & -0.1710 & -0.1170 \\
$0.4-0.5$ & 0.1788 & -0.0804 & -0.0782 \\
$0.5-0.6$ & 0.8329 & 0.3056 & 0.1936 \\
$0.6-0.7$ & 2.544 & 1.621 & 1.263 \\
$0.7-0.8$ & 6.914 & 5.468 & 4.557 \\
$0.8-0.9$ & 19.92 & 18.03 & 15.38 \\
\hline \hline
\end{tabular}

that will increase at low $Q^{2}$. More specifically we use [31]

$$
F_{i}^{H T}\left(x, Q^{2}\right)=F_{i}^{L T}\left(x, Q^{2}\right)\left(1+\frac{D_{i}(x)}{Q^{2}}\right)
$$

where $i=\{1,2,3\}$. We vary $D_{3}(x)$ over the range $[0,10] \mathrm{GeV}^{2}$ in steps of $1 \mathrm{GeV}^{2}$. (For the purposes of our simple illustration, it is sufficient to take $D_{3}(x)$ to be independent of $x$.) We will find that this range is well beyond what is allowed by experiment; note that even the first deviation, $D_{3}(x)=1$, lies well outside the allowed region of Table I. However, we display this exaggerated range to make the effect of the higher twist contributions evident.

To normalize this choice with the allowable range consistent with data, we compare with the MRST higher twist fit which extracted a limit on the function $D_{2}(x)$. We see from the table of $D_{2}(x)$ that the allowed contribution from the higher twist terms is quite small. In addition, we note that the sign for $D_{2}(x)$ obtained for the relevant small- $x$ region tends to be negative-exactly the opposite sign that is needed to move the theory toward the data.

As we have shown constraints on $D_{2}(x)$, the obvious question is should we expect $D_{3}(x)$ to be substantially different? A calculation of the power corrections using renormalons [32] suggests that while both $D_{1}(x)$ and $D_{3}(x)$ are of the same order of magnitude as $D_{2}(x)$ and have similar $x$-dependence, that $D_{1}(x)$ and $D_{3}(x)$ are even more negative than $D_{2}(x) ;^{7}$ again, this trend would move the theory farther from the data. Therefore, we conclude that $D_{2}(x)$ represents a conservative limit for the $D_{3}(x)$ power corrections.

Comparing the LO, NLO, and NNLO fits in Table I, we note a large variation, particularly in the $x$-range of interest

\footnotetext{
${ }^{7}$ This is generally supported by the analysis of CCFR data in Ref. [33].
}
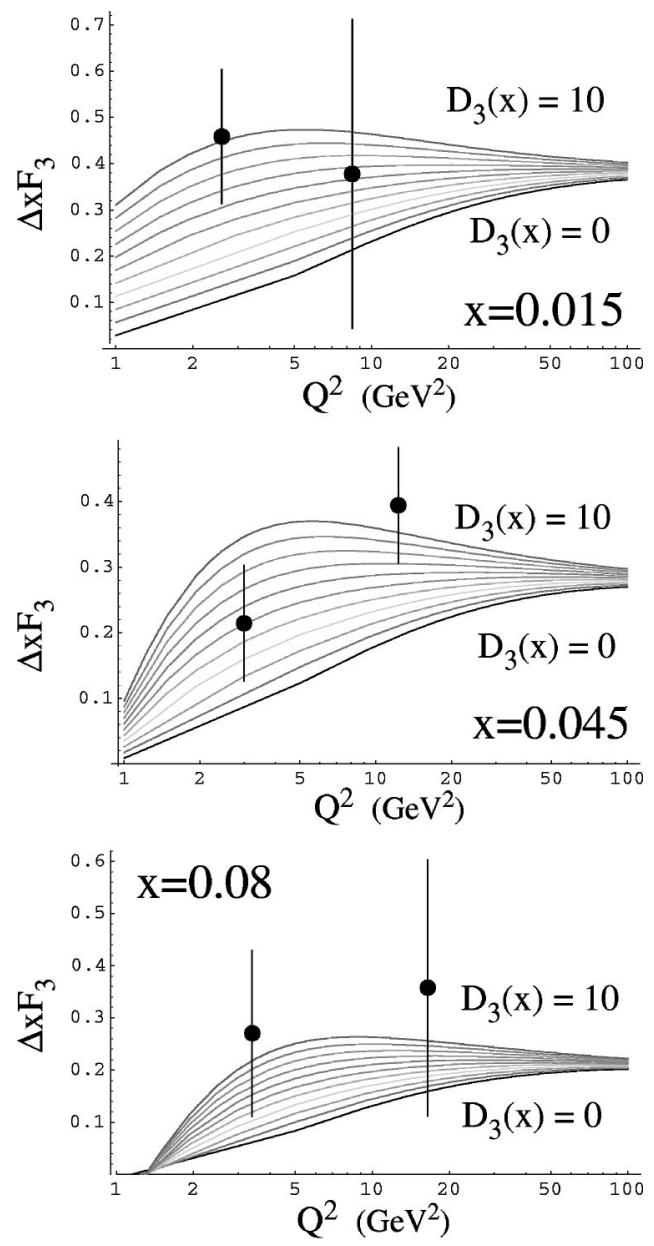

FIG. 7. Variation of the LO $\Delta x F_{3}$ on higher twist. The functional form is $\left[1+D_{3}(x) / Q^{2}\right]$ where we take $D_{3}(x)$ in the range $D_{3}(x)=[0,10] \mathrm{GeV}^{2}$ in steps of $1 \mathrm{GeV}^{2}$. Note that even the first deviation, $D_{3}(x)=1$, lies well outside the allowed region of Table I.

to us. The relative uncertainties of $D_{i}$ can be $100 \%$; however, this large relative uncertainty translates into a very small absolute uncertainty-as we shall see. Hence, one could ask whether correlated effects between $\left\{D_{1}, D_{2}, D_{3}\right\}$ might conspire to produce a magnified effect for $\Delta x F_{3}$. We will show that even this scenario is difficult given the constraints of Table I. For example, if we take $D_{i}=-0.23$, the largest higher-twist value in the $x$-range of interest, and allow ourselves to flip the sign so that these effects work in our favor, we find the factor $\left(1+D_{i}(x) / Q^{2}\right)$ results in only a $8 \%$ shift at the lowest $Q^{2}$ point. In a similar fashion, were two of the $D_{i}$ parameters to conspire to shift the theory curves, they would only be able to produce an effect of order $16 \%$. Whereas we need a shift in excess of $100 \%$ in order to move the theory curves toward the data, we see that adjusting the $D_{i}$ parameters yields no simple solution.

In summary, we observe from Fig. 7 that it would take an enormous higher twist contribution to bring the normalization of the theory curves in the range of the data points, and still the shape of the $Q^{2}$ dependence is not well matched; 
hence, we conclude that this is not a compelling solution.

\section{Isospin violations}

The naive parton model identity in Eq. (5) is modified if the full (non-diagonal) Cabibbo-Kobayashi-Maskawa (CKM) structure, NLO QCD radiative corrections, and QCD based charm production are taken into account. This expression is also modified even in leading-order if we have a violation of exact $p \leftrightarrow n$ isospin-symmetry (or charge symmetry); e.g., $u_{n}(x) \neq d_{p}(x)$. In deriving Eq. (5), isospinsymmetry was necessary to guarantee that the $u, d$-contributions cancel out in the difference, thereby leaving only the $s(x)$ and $c(x)$ contributions. ${ }^{8}$

The validity of exact charge symmetry (CS) has recently been reexamined [34]. Residual $u, d$-contributions to $\Delta x F_{3}$ from charge symmetry violation (CSV) would be amplified due to enhanced valence components $\left\{u_{v}(x), d_{v}(x)\right\}$, and because the $d \rightarrow u$ transitions are not subject to slow-rescaling corrections which strongly suppress the $s \rightarrow c$ contribution to $\Delta x F_{3}$, as illustrated in Fig. 4.

We will examine possible contributions to $\Delta x F_{3}$ by considering a "toy" model to parametrize the CS violations. In isospin space, we can parametrize a general transformation as a rotation:

$$
|q\rangle_{n}^{\mathrm{CSV}}=N_{q} \sum_{q^{\prime}} R_{q q^{\prime}}(\theta)\left|q^{\prime}\right\rangle_{p}
$$

where $R$ is a rotation matrix, and $N_{q}$ is the normalization factor. For example, in this model the $u$-distribution in the neutron would be related to the proton distributions via the relation:

$$
u_{n}^{\mathrm{CSV}}\left(x, Q^{2}\right)=N_{u}^{2}\left[\cos ^{2}(\theta) u_{p}\left(x, Q^{2}\right)+\sin ^{2}(\theta) d_{p}\left(x, Q^{2}\right)\right] .
$$

For $\theta=\pi / 2$, we recover the symmetric limit $u_{p}\left(x, Q^{2}\right)$ $=d_{n}\left(x, Q^{2}\right)$. Note that we define the normalization $N_{u}$ such that this model preserves the sum rule:

$$
\int d x u_{n}^{\mathrm{CSV}}\left(x, Q^{2}\right)-\bar{u}_{n}^{\mathrm{CSV}}\left(x, Q^{2}\right)=1 .
$$

Note that Eq. (10) should not be considered a serious theory, ${ }^{9}$ but rather a simple one-parameter $(\theta)$ model which is flexible enough to illustrate a range of CSV effects for $\Delta x F_{3}$.

In Fig. 8 we vary $\theta$ over the its maximum range $[0, \pi / 2]$ in steps of $\pi / 20$. The exact charge symmetry limit $\left(\theta_{0}\right.$ $=\pi / 2$ ) of Eq. (11) corresponds to the lowest curve in Fig. 8. Note the effect of the CSV contribution monotonically increases as $\theta$ deviates from the charge symmetry (CS) limit

\footnotetext{
${ }^{8}$ Note we have not investigated shadowing corrections. The CCFR data on $\mathrm{Fe}$ is converted with an isoscalar correction, and the corresponding uncertainties are included in the data [2].

${ }^{9}$ E.g., it does strictly speaking not commute with evolution.
}
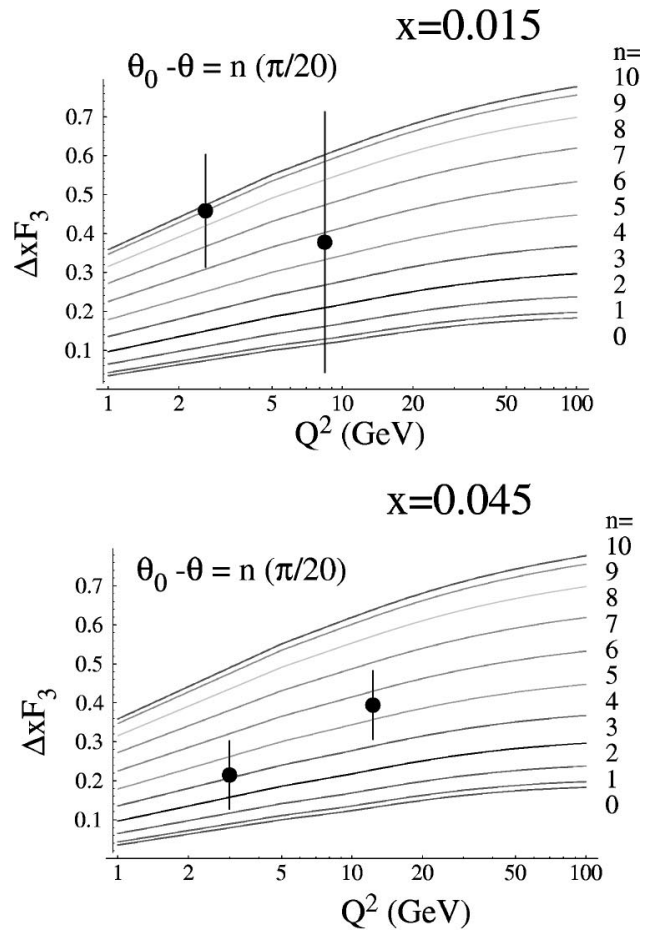

$\mathrm{x}=0.08$

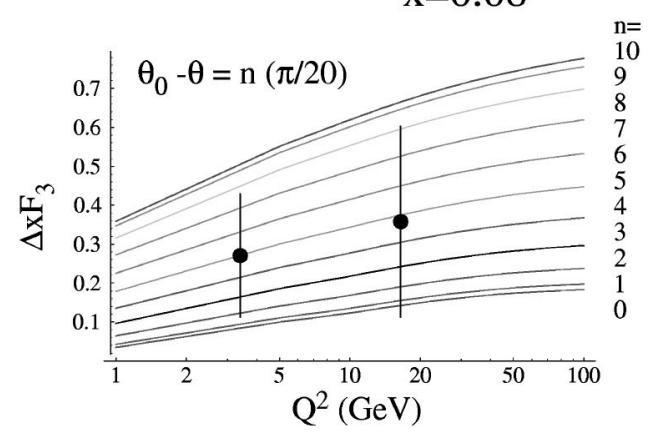

FIG. 8. Impact of a one-parametric charge symmetry violation (CSV) toy model of Eq. (10) and Eq. (11) on the LO $\Delta x F_{3}$ of Eq. (5). $\theta$ is varied over the range $\theta_{0}-\theta=[0, \pi / 2]$ in steps of $(\pi / 20)$.

$\theta_{0}=\pi / 2$. From the plot we observe that a violation of $\theta_{0}$ $-\theta \gtrsim(3 / 10)(\pi / 2)$ is required to bring the theory into the neighborhood of the data.

At the relevant $x$ values of interest for $\Delta x F_{3}$, this translates [via Eq. (11) and its analogue for $d_{n}$ ] into a $\sim 20 \%$ symmetry violation for $u_{n}$, and a $\sim 10 \%$ symmetry violation for $d_{n}$. Specifically,

$$
\begin{aligned}
& \frac{d_{p}(x)}{u_{n}^{\operatorname{CSV}}(x)} \simeq 1.2 \\
& \frac{u_{p}(x)}{d_{n}^{\operatorname{CSV}}(x)} \simeq 0.9 .
\end{aligned}
$$

Since the electroweak couplings are flavor-independent, $\left(V_{q}=A_{q}=1 \forall q\right), \Delta x F_{3}$ is in principle insensitive to a re- 


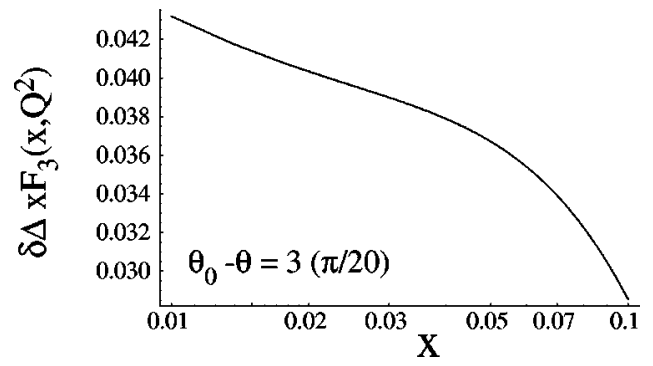

FIG. 9. $\delta \Delta x F_{3}\left(x, Q^{2}\right)$ with $Q^{2}=4 \mathrm{GeV}^{2}$ as defined in Eq. (15) and Eq. (11) for $\theta_{0}-\theta=3(\pi / 20)$.

shuffling of the CSV contributions between $u_{n}$ and $d_{n}$. In particular, if we define the shift due to CSV in $\Delta x F_{3}$ as $\delta \Delta x F_{3}$, we find

$$
\delta \Delta x F_{3} \equiv \frac{x}{4}(\delta d+\delta \bar{d}-\delta u-\delta \bar{u})
$$

where

$$
\delta u\left(x, Q^{2}\right) \equiv u_{n}^{\mathrm{CSV}}\left(x, Q^{2}\right)-d_{p}\left(x, Q^{2}\right) .
$$

The expression for $\delta \Delta x F_{3}\left(x, Q^{2}\right)$ of Eq. (15) is evaluated at $Q^{2}=4 \mathrm{GeV}^{2}$ from Eq. (11) with $\theta_{0}-\theta=(3 / 10)(\pi / 2)$ and is plotted in Fig. 9. ${ }^{10}$

Although this level of isospin violation certainly improves the description of $\Delta x F_{3}$, it is necessary to consider precisely what level of violation is actually allowed by other experimental data. For instance, it has previously been suggested that the discrepancy between $F_{2}$ from neutrino and muon data itself may be due to isospin violation [34]. This type of violation required that $\bar{u}$ be very different to $\bar{d}$ in the region of interest.

The measurement of the lepton charge asymmetry in $W$ decays from the Fermilab Tevatron $[35,36]$ places tight constraints on the up and down quark distributions in the range $0.007<x<0.24$, constraining them to be approximately as specified in the parton sets obtained by the global analyses. While only strictly telling us about parton distributions in the proton, this data rules out an isospin violation of this type to about $5 \%$ as demonstrated in [36].

However, there are other strong constraints on isospin violation. For example, we note that while the toy model above leaves the neutron singlet combination $q+\bar{q}$ invariant at the $\lesssim 2 \%$ level in the region $x \in[0.01 ; 0.1]$, it would lower the NC observable:

$$
\left.\left[\frac{4}{9}(u+\bar{u})+\frac{1}{9}(d+\bar{d})\right]\right|_{x \in[0.01 ; 0.1]}
$$

\footnotetext{
${ }^{10}$ Again, the detailed $x$-shape from Eq. (11) should not be taken as a serious model prediction.
}

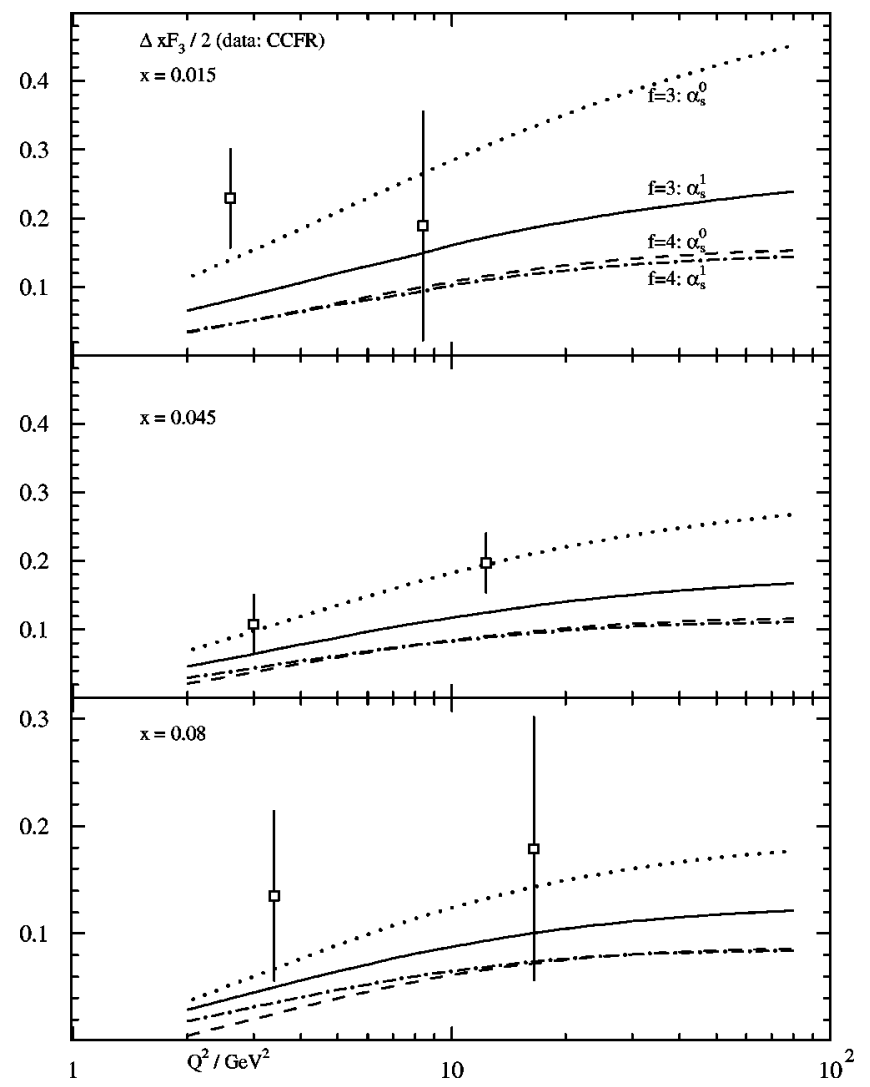

FIG. 10. LO and NLO calculations for $\Delta x F_{3} / 2$ vs $Q^{2}$ in 3 and 4 flavor schemes for three values of $x$. The dotted curve $\left(\mathrm{f}=3, \alpha_{s}^{0}\right)$ corresponds to the 3 -flavor LO result. The dashed curve $\left(\mathrm{f}=4, \alpha_{s}^{0}\right)$ corresponds to the 4 -flavor LO result. The solid and dot-dash curves correspond to the 3 and 4 flavor NLO QCD calculations, respectively.

by about $10 \%$. An effect of this size would definitely be visible in NMC $F_{2}^{n} / F_{2}^{p}$ data which has an uncertainty of order a few percent in this kinematic region, and acts as a major constraint [23].

At this point, one could play clever games to evade the constraints of specific experiments. For example, a reshuffling of CSV contributions between the individual $\delta q, \delta \bar{q}$ in Eq. (15) according to

$$
\begin{gathered}
x u_{n}=x d_{p}-\frac{2}{5} \delta \Delta x F_{3} \\
x d_{n}=x u_{p}+\frac{8}{5} \delta \Delta x F_{3} \\
x \bar{q}_{n} \text { :analogous }
\end{gathered}
$$

would keep Eq. (17) invariant. However, this would in turn raise the neutron singlet combination-as observed in $\mathrm{CC}$ DIS-by $\gtrsim 5 \%$; though it would help to explain the excess in $\delta x F_{3}$, it would spoil the new-found compatibility between neutral current and charged current data.

In addition, there are also fixed-target Drell-Yan experiments $[37,38]$ such as NA51 and E866 which precisely mea- 


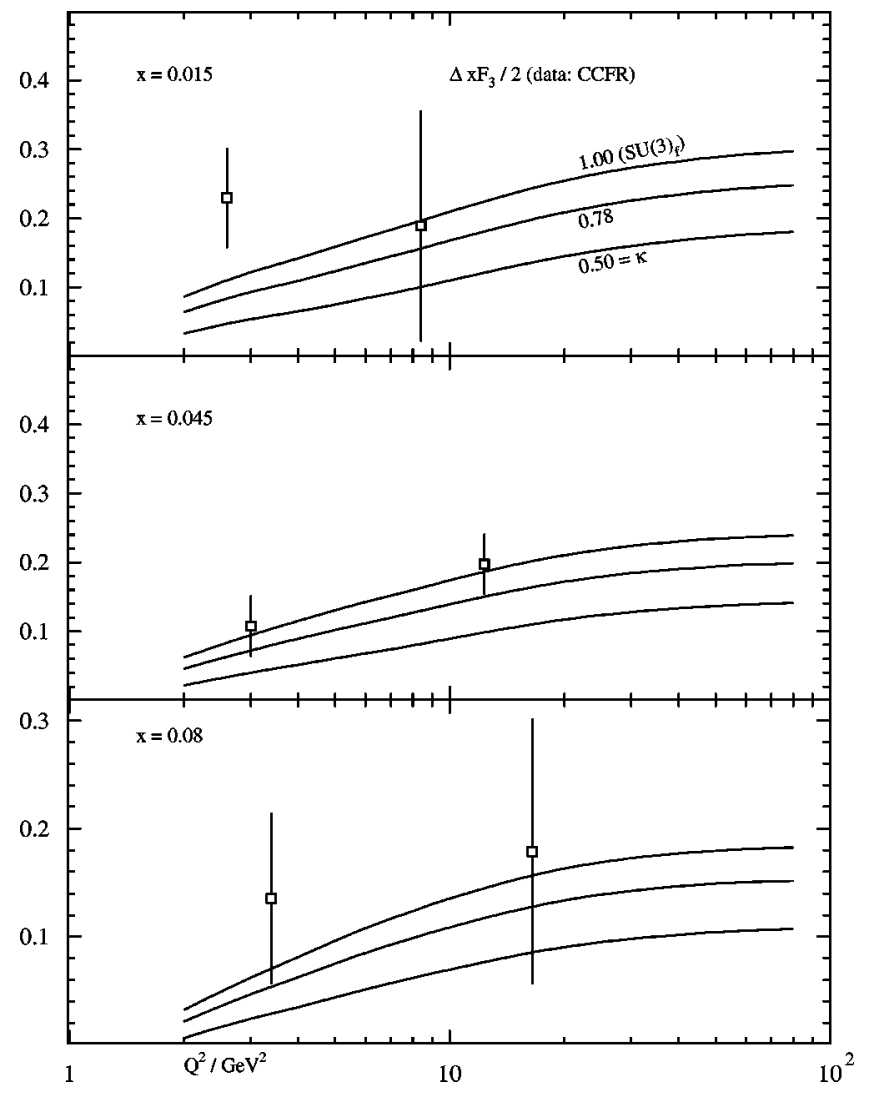

FIG. 11. Variation of $\Delta x F_{3} / 2$ on the strange-quark PDF. The NLO calculation is used with three sets of PDF's. These PDF sets are re-fit based on the MRST set, and the value of $\kappa$ is constrained to be $\kappa=\{0.50,0.78,1.00\}$.

sure $\bar{d} / \bar{u}$ in the range [38] $0.04<x<0.27$, and are also sensitive to isospin violating effects.

We therefore conclude that the many precise data sets which constrain different combinations of the PDF's probably leave no room for CSV contributions of the magnitude necessary to fully align the theory curves with the $\Delta x F_{3}$ data. However, we should add the caveat that an exhaustive investigation of the interplay of these different data sets and their influence on $\Delta x F_{3}$ will only be possible within a global PDF analysis

\section{NLO CALCULATION OF $\Delta x F_{3} / 2$}

Now that we have used the leading-order expression for $\Delta x F_{3}$ to systematically investigate dependence of this observable on various parameters, in this section we now turn to the full NLO calculation.

\section{A. Contributions to the NLO calculation}

In Fig. 10, we have plotted the LO and NLO calculations for $\Delta x F_{3}$ vs. $Q^{2}$ on an isoscalar target in 3 and 4 flavor schemes. The 3 -flavor LO calculation $\left(\mathrm{f}=3, \alpha_{s}^{0}\right)$ involves primarily the strange quark contribution, $s(x)$, as the charm distribution is excluded in this case. When the higher order terms are included $\left(\mathrm{f}=3, \alpha_{s}^{1}\right)$, this result moves (substan- tially) toward the predictions of the 4-flavor scheme. We note that while the 3 -flavor LO calculation $\left(\mathrm{f}=3, \alpha_{s}^{0}\right)$ appears consistent with the data, we cannot take this result as a precise theoretical prediction as this simplistic result is highly dependent on scheme and scale choices; a result that is verified by the large shift in going from LO to NLO.

The pair of curves in the 4-flavor scheme (using the CTEQ4HQ distributions) nicely illustrates how the charm distribution $c\left(x, \mu^{2}\right)$ evolves as $\ln \left(Q^{2} / m_{c}^{2}\right)$ for increasing $Q^{2}$; note, $c\left(x, \mu^{2}\right)$ enters with a negative sign so that the 4-flavor result is below the 3-flavor curve. For the scale choice, we take $\mu=\sqrt{Q^{2}+m_{c}^{2}}$. While the scale choice $\mu=Q$ is useful for instructive purposes such as demonstrating the matching of the 3- and 4-flavor calculations at $\mu=Q=m_{c}$, the choice $\mu=\sqrt{Q^{2}+m_{c}^{2}}$ is more practical as it provides a lower bound on $\mu$ which is important for the PDF's and $\alpha_{s}(\mu)$. (Cf., Sec. III C, and Ref. [39].)

Additionally we note the stability of the 4-flavor scheme in contrast to the 3-flavor scheme. The shift of the curves when including the NLO contributions is quite minimal, particularly when compared with the 3-flavor result. [40,41] This suggests that organizing the calculation to include the charm quark as a proton constituent can be advantageous even at relatively low values of the energy scale.

\section{B. PDF uncertainties: $s(x), \ldots$}

In Fig. 11, we show the variation of NLO calculation of $\Delta x F_{3}$ on the strange-quark PDF. To obtain a realistic assessment of the $s(x)$ dependence, we have use the NLO calculation with PDF's based on the MRST set which are re-fit with the value of $\kappa=2 s /(\bar{u}+\bar{d})$ constrained to be $\kappa$ $=\{0.50,0.78,1.00\}$. Note, by re-fitting the PDF's with the chosen value of $\kappa$ we are assured to have an internally consistent set of PDF's with appropriate matching between the quarks and gluon, and with the sum rules satisfied. ${ }^{11}$

The choice $\kappa=0.50$ is in line with the many experimental determinations of $\kappa$, cf. Table II; as expected, this prediction lies farthest from the data points.

The choice $\kappa=0.78$ is taken as an extreme upper limit given the experimental constraints; actually, in light of the results of Table II, this is arguably beyond present experimental bounds. This prediction is marginally consistent at the outer reach of the systematic + statistical error bars.

Finally, we take an $S U(3)$ symmetric set $(\kappa=1)$ purely for illustrative purposes. It is interesting to note that even this extreme value is still below the central value of the data points at the higher $x$ values.

In conclusion we note that increasing the strange quark distribution does succeed in moving the theory toward the

\footnotetext{
${ }^{11}$ Note, $\kappa$ is certainly $Q$-dependent, and the values for $\kappa$ quoted above correspond to the $Q_{0}$ of the evolution. While $\kappa$ compares the integral of $s(x)$ to the sea-quarks, there is also the possibility of an $x$-dependent variation [42]. This has been studied in the fits of the strange-sea $[11,15,12,43]$; we shall find that such subtle effects can play no role in resolving the $\Delta x F_{3}$ issue.
} 
TABLE II. Next-to-leading-order and leading-order fit results. Errors are statistical and systematic. This table is displayed to estimate the upper limits allowed by experiment; a full comparison must take into account scheme and scale choices, and the shape parameters.

\begin{tabular}{lccc}
\hline \hline Experiment & Order & $\kappa$ & Ref. \\
\hline CDHS & LO & $0.47 \pm 0.08 \pm 0.05$ & {$[10]$} \\
FMMF & LO & $0.41_{-0.075}^{+0.075}+0.063$ & {$[52]$} \\
CHARM II & LO & $0.39_{-0.06}^{+0.00}{ }_{-0.07}^{+0.07}$ & {$[13]$} \\
CCFR $^{\text {a }}$ & NLO & $0.477_{-0.044}^{+0.0046}+0.023$ & {$[15]$} \\
CCFR $^{\text {b }}$ & NLO & $0.468_{-0.046}^{+0.006}+0.024$ & {$[15]$} \\
CCFR $^{\text {b }}$ & LO & $0.373_{-0.041}^{+0.048} \pm 0.018$ & {$[11]$} \\
NOMAD & LO & $0.48_{-0.07}^{+0.09}+0.17$ & {$[14]$} \\
NuTeV & LO & $0.42^{+0.12} \pm 0.07 \pm 0.06$ & {$[12]$} \\
\hline \hline
\end{tabular}

${ }^{\mathrm{a}}$ Collins-Spiller fragmentation.

${ }^{\mathrm{b}}$ Peterson fragmentation.

data; however, our consistent NLO analysis presented here suggests that we have only limited freedom to increase $s(x)$, and that this alone is not sufficient to obtain good agreement between theory and data.

\section{Scheme choice}

In our final section, we present the best theoretical predictions presently available to demonstrate the scheme dependence of $\Delta x F_{3}(x, Q)$. Specifically, in Fig. 12 we show predictions for:

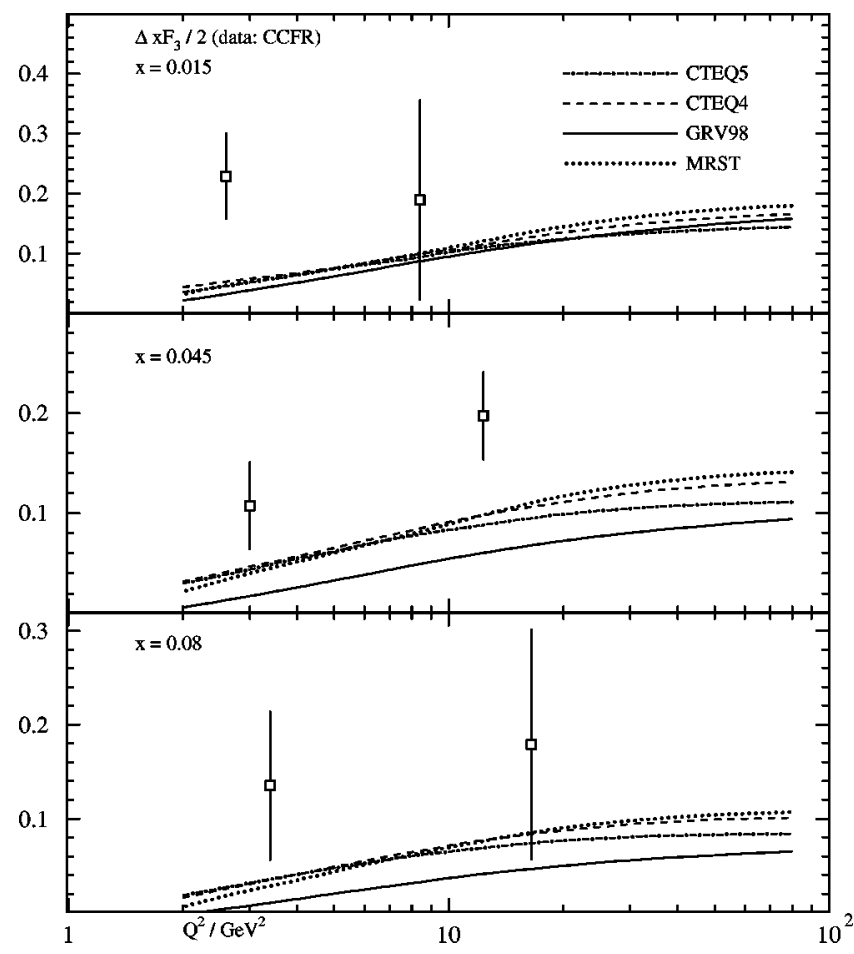

FIG. 12. Variation of $\Delta x F_{3} / 2$ on the renormalization scheme. All curves use NLO calculations, and appropriately matched PDF's. We note that the theoretical predictions are consistent within the theoretical uncertainty-as they should be.
NLO fixed flavor scheme (FFS) of Glück, Reya, and Vogt (GRV) [21].

NLO variable flavor number scheme (VFS) ThorneRoberts (TR) calculation [44].

NLO VFS ACOT calculation with CTEQ4 PDF's $[26,45,46]$.

NLO VFS ACOT calculation with CTEQ5 PDF's $[26,45,20]$.

All these calculations use NLO matrix elements, and are matched with appropriate global PDF's which are fitted in the proper scheme.

The first observation we make is how closely these four predictions match, especially given the wide variation displayed in previous plots such as Fig. 10. In hindsight, this result is simply a consequence of the fact that while different renormalization schemes can produce different results, this difference can only be higher order. ${ }^{12}$ Thus, the difference between these curves is indicative of terms of order $\alpha_{s}^{2}$ which have yet to be calculated. ${ }^{13}$ When terms of order $\alpha_{s}^{N}$ are included, the span of these predictions will be systematically reduced to order $\alpha_{s}^{N+1}$.

In Fig. 12, we note the very close agreement among the VFS calculations, particularly the TR calculation and the ACOT calculation with CTEQ4 PDF's. The ACOT calculation with the two CTEQ curves show primarily the effect of the charm distribution, as CTEQ4 uses $m_{c}=1.6$ and CTEQ5 uses $m_{c}=1.3$. The GRV calculation shows the effect of using yet a different scheme, in this case a FFN scheme, with its appropriately matched PDF. Were we to use MRST or CTEQ PDF's, the spread of these theory curves would decrease; however, this would most likely represent an underestimate of the true theoretical uncertainties arising from both the hard cross section and PDF's. ${ }^{14}$

While we consider it a triumph of QCD that different schemes truly yield comparable results (higher order terms aside), we should be cautious and note that the spread of these curves can only underestimate the true theoretical uncertainty. Note that GRV has a rather different strange distribution due to a different philosophy of obtaining this distribution rather than due to a different scheme.

\section{CONCLUSIONS AND OUTLOOK}

Comprehensive analysis of the neutrino data sets can provide incisive tests of the theoretical methods, particularly in the low $Q^{2}$ regime, and enable precise predictions that will facilitate new particle searches by constraining the PDF's. This document serves as a progress report, and work on these topics will continue in the future.

Theoretical predictions for $\Delta x F_{3}$ systematically undershoot preliminary fixed target data at the $\sim 1 \sigma$-level at low $x$

\footnotetext{
${ }^{12}$ To be precise, different renormalization schemes can differ by (i) terms of higher order in the perturbation series, and (ii) terms of higher twist which do not factorize [47,48].

${ }^{13}$ For asymptotic results at order $\alpha_{s}^{2}$, see Ref. [40].

${ }^{14}$ The computation of PDF errors is a complex subject. For some recent approaches to this topic see Refs. [19,20,49,43].
} 
and $Q$. The neutrino structure function $\Delta x F_{3}$ is obviously sensitive [50] to the strange sea of the nucleon and the details of deep inelastic charm production. A closer inspection reveals, however, considerable dependence upon factors such as the charm mass, factorization scale, higher twists, contributions from longitudinal $W^{ \pm}$polarization states, nuclear shadowing, charge symmetry violation, and the PDF's. This makes $\Delta x F_{3}\left(x, Q^{2}\right)$ an excellent tool to probe both perturbative and non-perturbative QCD.

We have explored the variation of $\Delta x F_{3}\left(x, Q^{2}\right)$ on the above factors and found none of these to be capable of resolving the discrepancy between the data and theory.

Although we have not eliminated the possibility that separate effects conspire to align the theory with the data, we have demonstrated this to be extremely unlikely. Of course, a definitive answer can only be obtained by a global analysis which combines the neutrino data for dimuons, $\Delta x F_{3}$, $F_{2}^{\nu}(P M I)$, and $F_{2}^{\mu, e}$.
As the situation stands now, this $\Delta x F_{3}\left(x, Q^{2}\right)$ puzzle poses an important challenge to our understanding of QCD and the related nuclear processes in an important kinematic region. The resolution of this puzzle is important for future data analysis, and the solution is sure to be enlightening, and allow us to expand the applicable regime of the QCD theory.

\section{ACKNOWLEDGMENTS}

This work is supported by the Royal Society, the U.S. Department of Energy, the National Science Foundation, the Lightner-Sams Foundation, and the "Bundesministerium für Bildung, Wissenschaft, Forschung und Technologie," Bonn. R.S.T. would like to thank the Royal Society for financial support. We thank J. Bluemlein, A. Bodek, J. Conrad, J. Morfin, S. Kuhlmann, R.G. Roberts, H. Schellman, M. Shaevitz, J. Smith, and W.-K. Tung for valuable discussions.
[1] CCFR-NuTeV Collaboration, U.K. Yang et al., Phys. Rev. Lett. 86, 2792 (2001).

[2] CCFR Collaboration, W.G. Seligman et al., Phys. Rev. Lett. 79, 1213 (1997); W. G. Seligman, Ph.D. thesis, Columbia University, Nevis-292, 1997.

[3] CCFR-NuTeV Collaboration, B.T. Fleming et al., hep-ex/0011095.

[4] L.W. Whitlow, E.M. Riordan, S. Dasu, S. Rock, and A. Bodek, Phys. Lett. B 282, 475 (1992).

[5] C. Boros, F.M. Steffens, J.T. Londergan, and A.W. Thomas, Phys. Lett. B 468, 161 (1999).

[6] For the discussion dealing with the extraction of $x(s-c)$ using charged and neutral current data the possibilities to measure $\Delta x F_{3}$ at the DESY ep collider HERA, cf. J. Blumlein, Surv. High Energy Phys. 7, 181 (1994); J. Blumlein, M. Klein, T. Naumann, and T. Riemann, PHE-88-01, Contribution to Proc. of DESY Theory Workshop on Physics at HERA, Hamburg, West Germany, 1987.

[7] W.T. Giele, S. Keller, and E. Laenen, Nucl. Phys. B (Proc. Suppl.) 51C, 255 (1996); Phys. Lett. B 372, 141 (1996); hep-ph/9408325.

[8] R. Demina et al., hep-ph/0005112; L. de Barbaro et al., hep-ph/0006300.

[9] R.D. Ball, D.A. Harris, and K.S. McFarland, hep-ph/0009223.

[10] CDHSW Collaboration, H. Abramowicz et al., Z. Phys. C 15, 19 (1982)

[11] CCFR Collaboration, S.A. Rabinowitz et al., Phys. Rev. Lett. 70, 134 (1993).

[12] NuTeV Collaboration, T. Adams et al., hep-ex/9906037.

[13] CHARM II Collaboration, P. Vilain et al., Eur. Phys. J. C 11, 19 (1999)

[14] NOMAD Collaboration, P. Astier et al., Phys. Lett. B 486, 35 (2000).

[15] CCFR Collaboration, A. O. Bazarko et al., Z. Phys. C 65, 189 (1995); A. O. Bazarko, Ph.D. thesis, NEVIS-1504.

[16] NuTeV Collaboration, M. Goncharov et al., hep-ex/0102049.
[17] M. Glück, S. Kretzer, and E. Reya, Phys. Lett. B 398, 381 (1997); 405, 392(E) (1997).

[18] S. Kretzer and F. Olness (in preparation).

[19] A.D. Martin, R.G. Roberts, W.J. Stirling, and R.S. Thorne, Eur. Phys. J. C 14, 133 (2000).

[20] H.L. Lai et al., Eur. Phys. J. C 12, 375 (2000).

[21] M. Glück, E. Reya, and A. Vogt, Eur. Phys. J. C 5, 461 (1998).

[22] CTEQ1 Collaboration, J. Botts et al., Phys. Lett. B 304, 159 (1993).

[23] NMC Collaboration, M. Arneodo et al., Nucl. Phys. B483, 3 (1997).

[24] A.I. Signal and A.W. Thomas, Phys. Lett. B 191, 205 (1987); S.J. Brodsky and B.-Q. Ma, ibid. 381, 317 (1996).

[25] R.M. Barnett, Phys. Rev. Lett. 36, 1163 (1976); H. Georgi and H.D. Politzer, Phys. Rev. D 14, 1829 (1976).

[26] F. Olness and W.K. Tung, Nucl. Phys. B308, 813 (1988); M. Aivazis, F. Olness, and W.K. Tung, Phys. Rev. D 50, 3085 (1994); M. Aivazis, J.C. Collins, F. Olness, and W.K. Tung, ibid. 50, 3102 (1994).

[27] T. Gottschalk, Phys. Rev. D 23, 56 (1981); M. Glück, S. Kretzer, and E. Reya, Phys. Lett. B 380, 171 (1996); 405, 391(E) (1996).

[28] ZEUS Collaboration, J. Breitweg et al., Eur. Phys. J. C 12, 35 (2000); H1 and ZEUS Collaborations, C. Coldewey, Nucl. Phys. B (Proc. Suppl.) 74, 209 (1999).

[29] Particle Data Group, D.E. Groom et al., Eur. Phys. J. C 15, 1 (2000), available on the PDG WWW pages (URL: http:// pdg.lbl.gov/).

[30] J.C. Collins, ANL-HEP-CP-90-58, Proc. of 25th Rencontre de Moriond: High Energy Hadronic Interactions, Les Arcs, France, 1990.

[31] A.D. Martin, R.G. Roberts, W.J. Stirling, and R.S. Thorne, Phys. Lett. B 443, 301 (1998).

[32] M. Dasgupta and B.R. Webber, Phys. Lett. B 382, 273 (1996).

[33] A.L. Kataev, A.V. Kotikov, G. Parente, and A.V. Sidorov, Phys. Lett. B 417, 374 (1998); S.I. Alekhin and A.L. Kataev, ibid. 452, 402 (1999); A.L. Kataev, G. Parente, and A.V. 
Sidorov, Nucl. Phys. B573, 405 (2000).

[34] C. Boros, J.T. Londergan, and A.W. Thomas, Phys. Rev. D 59, 074021 (1999); Phys. Rev. Lett. 81, 4075 (1998), and related references therein.

[35] CDF Collaboration, F. Abe et al., Phys. Rev. Lett. 81, 5754 (1998).

[36] A. Bodek, Q. Fan, M. Lancaster, K.S. McFarland, and U.K. Yang, Phys. Rev. Lett. 83, 2892 (1999).

[37] NA51 Collaboration, A. Baldit et al., Phys. Lett. B 332, 244 (1994).

[38] FNAL E866/NuSea Collaboration, E.A. Hawker et al., Phys. Rev. Lett. 80, 3715 (1998).

[39] C. Schmidt, hep-ph/9706496; J. Amundson, C. Schmidt, W.K. Tung, and X. Wang, J. High Energy Phys. 10, 031 (2000); J. Amundson, F. Olness, C. Schmidt, W.K. Tung, and X. Wang, FERMILAB-CONF-98-153-T, 1998.

[40] M. Buza and W.L. van Neerven, Nucl. Phys. B500, 301 (1997); M. Buza, Y. Matiounine, J. Smith, R. Migneron, and W.L. van Neerven, ibid. B472, 611 (1996).

[41] A. Chuvakin, J. Smith, and W.L. van Neerven, Phys. Rev. D 61, 096004 (2000); 62, 036004 (2000); A. Chuvakin and J. Smith, ibid. 61, 114018 (2000).
[42] J. Ellis, Nucl. Phys. A684, 53 (2001).

[43] V. Barone, C. Pascaud, and F. Zomer, Eur. Phys. J. C 12, 243 (2000).

[44] R.S. Thorne and R.G. Roberts, hep-ph/0010344; Phys. Rev. D 57, 6871 (1998).

[45] S. Kretzer and I. Schienbein, Phys. Rev. D 56, 1804 (1997); 58, 094035 (1998); 59, 054004 (1999).

[46] H.L. Lai et al., Phys. Rev. D 55, 1280 (1997).

[47] J.C. Collins and W.-K. Tung, Nucl. Phys. B278, 934 (1986); J.C. Collins, Phys. Rev. D 58, 094002 (1998).

[48] M. Krämer, F. Olness, and D. Soper, Phys. Rev. D 62, 096007 (2000).

[49] S. Alekhin, Eur. Phys. J. C 10, 395 (1999); W.T. Giele and S. Keller, Phys. Rev. D 58, 094023 (1998); W.T. Giele, S. Keller, and D.A. Kosower, hep-ph/0104052; J. Pumplin, D.R. Stump, and W.K. Tung, hep-ph/0008191.

[50] V. Barone, U. D’Alesio, and M. Genovese, Phys. Lett. B 357, 435 (1995).

[51] A.D. Martin, R.G. Roberts, W.J. Stirling, and R.S. Thorne, Eur. Phys. J. C 18, 117 (2000).

[52] B. Strongin et al., Phys. Rev. D 43, 2778 (1991). 